
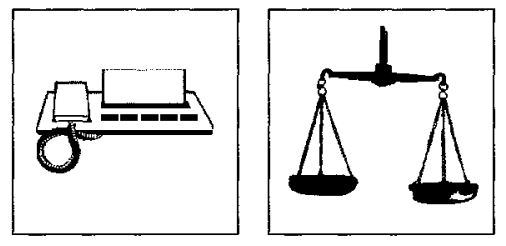

\title{
Die Übermittlung
}

fristwahrender Schriftsätze per Telefax

\author{
BVerf.G, Beschluß vom 1. August 1996 (1 BvR 121/95) - "Fristwabrung per Telefax I"
}

(Verfassungsbeschwerde der W:-GmbH i. L. gegen das Urteil des Bundesarbeitsgerichts vom 14. September 1994 (2 AZR 95/94) sowie gegen die Urteile des Landesarbeitsgerichts Mecklenburg-Vorpommern vom 7. Oktober 1993 (1 Sa 4/93, $1 \mathrm{Sa}$ 5/93, $1 \mathrm{Sa}$ 6/93, $1 \mathrm{Sa}$ 28/93 und $1 \mathrm{Sa}$ 29/93.)

\section{Leitsätze der Redaktion}

1. Die Übermittlung fristwahrender Schriftsätze per Telefax ist in allen Gerichtszweigen uneingeschränkt zulässig.

2. Der Zugang eines Telefaxes ist zu fingieren, wenn Anhaltspunkte dafür vorliegen, daß die abgesandten Signale eingegangen sind, das Empfangsgerät daraus aber keinen vollständigen Ausdruck gefertigt hat.

3. Auf den Bürger darf nicht die Verantwortung für Risiken und Unsicherheiten bei der Entgegennahme rechtzeitig in den Gewahrsam des Gerichts gelangter, fristwahrender Schriftsätze abgewälz $\dagger$ werden, sofern die Ursache hierfür allein in der Sphäre des Gerichts zu finden ist.

4. Die aus den technischen Gegebenheiten eines vom Gericht eröffneten Kommunikationsmittels herrührenden besonderen Risiken dürfen nicht auf den Nutzer dieses Mediums abgewälzt werden. Dies gilt sowohl für Störungen des Empfangsgeräts im Gericht als auch für Störungen der Übermittlungsleifungen.

5. Der Nutzer hat mit der Wahl eines anerkannten Übermittlungsmediums, der ordnungsgemäßen Nutzung eines funktionsfähigen Sendegeräts und der korrekten Eingabe der Empfängernummer das seinerseits Erforderliche zur Fristwahrung getan, wenn er so rechtzeitig mit der Übermittlung beginnt, daß unter normalen Umständen mit ihrem AbschluB bis 24.00 Uhr zu rechnen ist.

\section{Entscheidungsformel}

Einstimmig beschlossen: Art. 21 GG und Rechtsstaatsprinzip sind verletzt.

Zurückverweisung

Wiedereinsetzung versagt

Verfristete

Berufungsbegründung per

Telefax

$L A G$ :

Ausnutzung des Fristendes begründet erböbte Sorgfaltspflicht.
Das Urteil des Bundesarbeitsgerichts vom 14. September 1994 - 2 AZR 95/94 - und die Urteile des Landesarbeitsgerichts Mecklenburg-Vorpommern vom 7. Oktober 1993 - 1 Sa 4/93, 1 Sa 5/93, 1 Sa 6/93, 1 Sa 28/93 und 1 Sa 29/93 - verletzen die Beschwerdeführerin in ihrem Recht aus Artikel 2 Absatz 1 des Grundgesetzes in Verbindung mit dem Rechtsstaatsprinzip. Sie werden aufgehoben.

Die Sachen werden an das Landesarbeitsgericht Mecklenburg-Vorpommern zurückverwiesen. Das Land Mecklenburg-Vorpommern hat der Beschwerdeführerin die notwendigen Auslagen zu erstatten.

\section{Gründe}

A.

Die Verfassungsbeschwerde richtet sich gegen die Versagung der Wiedereinsetzung in den vorigen Stand, nachdem die Übermittlung fristwahrender Schriftsätze per Telefax gescheitert war. I.

1. Die Beschwerdeführerin, eine $\mathrm{GmbH}$ in Liquidation, unterlag in fünf Kündigungsschutzverfahren vor dem Arbeitsgericht Schwerin. Ihr Liquidator ist als Rechtsanwalt in einer Anwaltskanzlei tätig, die ein $Z$ weitbüro am Sitz des zuständigen Landesarbeitsgerichts in Rostock hat. Zweitinstanzlich ließ sich die Beschwerdeführerin durch einen Prozeßbevollmächtigten aus Hamburg vertreten. Dessen Berufungsbegründungen gingen erst einen Tag nach Ablauf der verlängerten Begründungsfrist beim Landesarbeitsgericht ein. Die Beschwerdeführerin beantragte fristgerecht Wiedereinsetzung in den vorigen Stand. Am letzten Tag der Begründungsfrist sei bis 20.31 Uhr vergeblich versucht worden, die jeweilige Begründung per Fax von Hamburg dem Gericht in Rostock zu übermitteln. Es sei nicht zu klären, ob dies auf einem Defekt oder Papiermangel beim Empfangsgerät des Landesarbeitsgerichts oder auf einer Leitungsstörung beruhe. Bei einer Telefonkontrolle der Fax-Leitung sei das Freizeichen ertönt.

2. Das Landesarbeitsgericht verwarf die Berufungen als unzulässig, ohne zu klären, worauf die gescheiterte Übermittlung per Fax zurückzuführen war. Der Beschwerdeführerin sei die beantragte Wiedereinsetzung in den vorigen Stand zu versagen. Unter Berücksichtigung der durch die Ausnutzung der Frist bis zum letzten Tag entstandenen erhöhten Sorgfaltspflicht hätten die Übermittlungsbemühungen nicht um 20.31 Uhr abgebrochen werden dürfen. Der Prozeßbevollmächtigte der Beschwerdeführerin habe vielmehr eine andere Vermittlungs- 
möglichkeit suchen müssen. Auch wenn ihm eine Fahrt nach Rostock nicht zumutbar gewesen sei, hätte ein in den Nachtbriefkasten des Gerichts einzuwerfendes Blitztelegramm aufgegeben werden können. Auch hätten die Berufungsbegründungen per Fax einem anderen Rechtsanwalt in Rostock übermittelt werden können, damit dieser sie in den Nachtbriefkasten werfe. Dies sei hier um so naheliegender gewesen, als die Kanzlei des Liquidators der Beschwerdeführerin in Rostock ein Zweitbüro unterhalte, das über einen Faxanschluß verfüge.

Die Revisionen wurden vom Bundesarbeitsgericht zur gemeinsamen Entscheidung verbunden und zurückgewiesen. Das Landesarbeitsgericht habe dargestellt, welche zumutbaren Wege der fristgerechten Übermittlung bis Mitternacht es noch gegeben habe. Telegrafische Begründungen hätten eventuell auf den formal notwendigen Inhalt gekürzt werden können. Möglicherweise habe es beim Landesarbeitsgericht auch einen Nachtportier gegeben, den der Prozeßbevollmächtigte der Beschwerdeführerin telefonisch hätte erreichen und der eine Störung des Empfangsgeräts hätte beseitigen können. Die Beschwerdeführerin habe nicht vorgetragen, daß derartige Möglichkeiten nicht bestanden hätten.

II.

Mit ihrer Verfassungsbeschwerde macht die Beschwerdeführerin geltend, sie habe darauf vertrauen dürfen, daß die von den Gerichten zur Verfügung gestellten Kommunikationseinrichtungen auch funktionsfähig seien. Die Kosten eines Blitztelegramms hätten in keinem Verhältnis zur Bedeutung des Prozesses gestanden. Außerdem würden Telegramme in Rostock nach 20.30 Uhr nicht mehr zugestellt. Ein Pförtner könne nach Dienstschluß nicht mehr telefonisch erreicht werden. Er wäre gegebenenfalls auch weder befugt noch fähig, ein defektes Telefaxgerät zu reparieren.

Außerdem sei Art. 101 Abs. 1 Satz 2 GG verletzt. Das Bundesarbeitsgericht habe die Rechtssache dem Gemeinsamen Senat der obersten Gerichtshöfe vorlegen müssen. Es sei von der Entscheidung des Bundesgerichtshofs vom 2. Oktober 1991 (NJW 1992, S. 244) abgewichen. Dieser habe verlangt, daß die Justizbehörden auch nach Dienstschluß für die Funktionsfähigkeit eines Telefaxgeräts zu sorgen hätten, wenn sie den Zugang zum Gericht durch ein solches Gerät eröffnet hätten.

III.

Zu der Verfassungsbeschwerde hat der Präsident des Bundesgerichtshofs Stellung genommen.

Vertrauen in funktionsfäbige

Kommunikationsgeräte der Gerichte

$B A \mathrm{G}$ :

Zumutbare Alternativen

fristgerechter Übermittlung

Abweichung des $B A \mathrm{G}$ von

BGH NJW 1992, S. 244

B.

I.

Die Verfassungsbeschwerde ist zulässig. Sie ist innerhalb der Frist des $₫ 93$ Abs. 1 Satz 1 BVerfGG eingelegt worden. Allerdings sind die letzte Seite der Beschwerdeschrift mit der Unterschrift des Prozeßbevollmächtigten der Beschwerdeführerin sowie die beigefügten Anlagen erst nach Fristablauf ausgedruckt worden. Die ankommenden Signale sind jedoch von einem Telefaxgerät des Bundesverfassungsgerichts, das einen Internspeicher besitzt, noch am letzten Tag der Frist empfangen worden. Dies ergibt sich aus einem entsprechenden Vermerk auf dem Fax-Ausdruck. Der Zugang eines Telefaxes ist zu fingieren, wenn Anhaltspunkte dafür vorliegen, daß die abgesandten Signale eingegangen sind, das Empfangsgerät daraus aber keinen vollständigen Ausdruck gefertigt hat (so schon für den Bereich der Zivilprozeßordnung: BGHZ 105, 40 [44 f.]; NJW 1994, S. 1881 [1882]) . Die Verfassungsbeschwerde ist damit fristgerecht eingegangen.

II.

Die Annahme der Verfassungsbeschwerde ist zur Durchsetzung der in $\mathbb{9} 90$ Abs. 1 BVerfGG genannten Rechte angezeigt ( $\$ 93$ a Abs. 2 Buchstabe b BVerfGG). Die Beschwerdeführerin ist offensichtlich in ihrem Grundrecht auf effektiven Rechtsschutz (Art. 2 Abs. 1 GG in Verbindung mit dem Rechtsstaatsprinzip) verletzt. Das Bundesverfassungsgericht hat die für die Beurteilung der Verfassungsbeschwerde maßgeblichen verfassungsrechtlichen Fragen bereits geklärt ( $\$ 93$ c Abs. 1 Satz 1 BVerfGG).

1. Der Anspruch auf Gewährung wirkungsvollen Rechtsschutzes (Art. 2 Abs. 1 GG in Verbindung mit dem Rechtsstaatsprinzip) verbietet es den Gerichten, den Parteien den Zugang $\mathrm{zu}$ einer in der Verfahrensordnung eingeräumten Instanz in unzumutbarer, aus Sachgründen nicht mehr zu rechtfertigender Weise zu erschweren (BVerfGE 69, 381 [385], stRspr; zuletzt BVerfGE 88, 118 [123 ff.]). Die Gerichte dürfen daher bei Auslegung der die Wiedereinsetzung in den vorigen Stand regelnden Vorschriften die Anforderungen an das, was der Betroffene veranlaßt haben muß, um Wiedereinsetzung zu erlangen, nicht überspannen (BVerfGE 40, 88 [91]; 67, 208 [212 f.]; stRspr). Allerdings sind die nach der jeweiligen prozessualen Lage gegebenen und zumutbaren Anstrengungen zur Wahrung des rechtlichen Gehörs zu verlangen (BVerfGE 74, 220 [225]). Etwaige Fristversäumnisse, die auf der Verzöge-

Annabme zur Entscheidung

Zulässige

Verfassungsbescbwerde bei

Fiktion rechtzeitigen Zugangs

Wiedereinsetzung darf.nicht in unzumutbarer Weise erscbwert werden. 


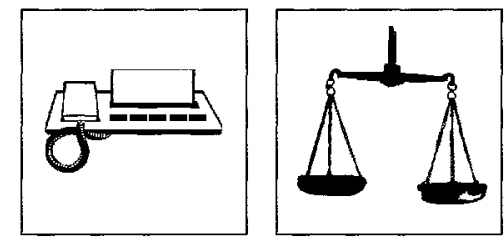

Die angegriffenen

Entscheidungen setzen Sorgfaltsmaßstab zu hoch an.

Anstrengungen des Prozeßvertreters waren ausreichend.

Schriftsatzverkebriper Fax ist zulässig.

Risiken des Telefaxverkebrs nicbt auf.Nutzer abwälzbar

Sorgfaltspflicht $=$ ordnungsgemäße Nutzung + korrekte Nummernwabl + erwartetes Übermittlungsende vor $24 \mathrm{Ubr}$

Verzögerung stellt keine unangemesse

Verfabrensverzögerung dar.

Verletzung des allgemeinen Gleichbeitssatzes

Aufbebung und Zurïckverweisung rung der Entgegennahme von Schriftsätzen durch das Gericht beruhen, dürfen dem Bürger aber nicht angelastet werden (BVerfGE 52, 203 [207] m.w.N.) . Ebensowenig darf auf ihn die Verantwortung für Risiken und Unsicherheiten bei der Entgegennahme rechtzeitig in den Gewahrsam des Gerichts gelangter fristwahrender Schriftsätze abgewälzt werden, sofern die Ursache hierfür allein in der Sphäre des Gerichts zu finden ist (BVerfGE 69, 381 [386]).

2. Diesem verfassungsrechtlichen Maßstab werden die angegriffenen Entscheidungen nicht gerecht. Sie überspannen die vom Prozeßbevollmächtigten der Beschwerdeführerin zu erfüllenden Sorgfaltspflichten und wälzen Risiken bei der Benutzung eines Faxgerätes, die allein in der Sphäre des Gerichts liegen, auf den rechtsuchenden Bürger ab.

Das Landesarbeitsgericht hat nicht geklärt, ob die Übermittlung per Telefax wegen Leitungsstörungen oder wegen eines Fehlers am Gerät des Landesarbeitsgerichts gescheitert ist. Das Scheitern war jedenfalls nicht auf eine Überlastung der Leitungen zurückzuführen. Dies ergibt sich aus dem unbestrittenen Vortrag des Prozeßbevollmächtigten der Beschwerdeführerin, bei einem Kontrollanruf sei ein Freizeichen ertönt. In beiden denkbaren Störfällen durften die Gerichte jedoch keine weiteren Anstrengungen des Prozeßbevollmächtigten der Beschwerdeführerin zur fristgerechten Übermittlung der Berufungsbegründungen verlangen.

Die Übermittlung fristwahrender Schriftsätze per Telefax ist in allen Gerichtszweigen uneingeschränkt zulässig (Hoppmann, VersR 1992, S. 1068 [Fn 6 m. w. N.]; s. auch BVerfG, 2. Kammer des Zweiten Senats, NJW-RR 1995, S. 441 [442]).

Wird dieser Übermittlungsweg durch ein Gericht eröffnet, so dürfen die aus den technischen Gegebenheiten dieses Kommunikationsmittels herrührenden besonderen Risiken nicht auf den Nutzer dieses Mediums abgewälzt werden. Dies gilt im besonderen für Störungen des Empfangsgeräts im Gericht. In diesem Fall liegt die entscheidende Ursache für die Fristsäumnis in der Sphäre des Gerichts. Aber auch Störungen der Übermittlungsleitungen sind dem gewählten Übermittlungsmedium immanent, da ein Telefax nur über sie zum Empfangsgerät gelangt. Erst Leitungen und Gerät gemeinsam stellen die vom Gericht eröffnete Zugangsmöglichkeit dar. Auch bei einer Leitungsstörung versagt daher die von der Justiz angebotene Zugangseinrichtung.

Der Nutzer hat mit der Wahl eines anerkannten Übermittlungsmediums, der ordnungsgemäßen Nutzung eines funktionsfähigen Sendegeräts und der korrekten Eingabe der Empfängernummer das seinerseits Erforderliche zur Fristwahrung getan, wenn er so rechtzeitig mit der Übermittlung beginnt, daß unter normalen Umständen mit ihrem Abschluß bis 24.00 Uhr zu rechnen ist.

Von einem Rechtsanwalt, der sich und seine organisatorischen Vorkehrungen darauf eingerichtet hat, einen Schriftsatz weder selbst noch durch Boten oder per Post, sondern durch Fax zu übermitteln, kann daher beim Scheitern der gewählten Übermittlung infolge eines Defekts des Empfangsgeräts oder wegen Leitungsstörungen nicht verlangt werden, daß er innerhalb kürzester Zeit eine andere als die gewählte, vom Gericht offiziell eröffnete Zugangsart sicherstellt. Fristen sollen die Gerichte vor unangemessenen Verfahrensverzögerungen schützen (vgl. BVerfGE 88, 118 [124]). Eine Verzögerung, die allein infolge eines in der Sphäre des Gerichts liegenden Umstandes eintritt, kann in diesem Sinne nicht als unangemessen betrachtet werden. Die den angegriffenen Entscheidungen zugrundeliegende Auffassung (ähnlich: BGH, NJW 1992, S. 244; NJW 1995, S. 1431 [1432]; BAGE 65, 255 [259]; BSG, AP Nr. 26 zu $₫ 233$ ZPO 1977; OLG München, VersR 1991, S. 831) führt zudem zu einer Ungleichbehandlung vergleichbarer Sachverhalte: Ein Prozeßbevollmächtigter, der seinen Schriftsatz bereits am frühen oder späten Nachmittag des letzten Tages der Frist fertiggestellt hat, müßte danach beim Scheitern einer Übermittlung per Telefax unter erheblichem Zeit- und Kostenaufwand alle nur denkbaren Anstrengungen unternehmen, um den fristgerechten Eingang bei Gericht doch noch sicherzustellen. Demgegenüber müßte ein Anwalt, der seinen Schriftsatz erst kurz vor Fristablauf fertigt, ohne weiteres Wiedereinsetzung erhalten, sofern er nur einen fehlgeschlagenen Übermittlungsversuch so zeitig begonnen hat, daß er unter normalen Umständen bis 24.00 Uhr abgeschlossen worden wäre (ähnlich BVerfGE 52, 203 [211 f.]).

III.

Die angegriffenen Entscheidungen sind aufzuheben und die Rechtsstreitigkeiten an das Landesarbeitsgericht Mecklenburg-Vorpommern zurückzuverweisen ( $\$ 95 \mathrm{Abs}$. 2 BVerfGG).

Das Land Mecklenburg-Vorpommern hat die notwendigen Auslagen der Beschwerdeführerin zu erstatten ( $\$ 34$ a Abs. 2 BVerfGG).

Kübling Jaeger Steiner

Eingesandt von Ass. jur. Jens M. Schmittmann, Essen. 